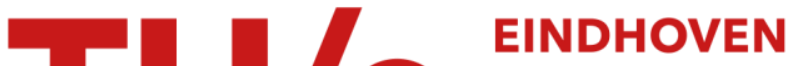 UNIVERSITY OF TECHNOLOGY
}

\section{Effect of bending flexibility on the phase behavior and dynamics of rods}

\section{Citation for published version (APA):}

Naderi, M., \& Schoot, van der, P. P. A. M. (2014). Effect of bending flexibility on the phase behavior and dynamics of rods. Journal of Chemical Physics, 141, 1-10. [124901]. https://doi.org/10.1063/1.4895730

DOI:

$10.1063 / 1.4895730$

Document status and date:

Published: 01/01/2014

\section{Document Version:}

Publisher's PDF, also known as Version of Record (includes final page, issue and volume numbers)

\section{Please check the document version of this publication:}

- A submitted manuscript is the version of the article upon submission and before peer-review. There can be important differences between the submitted version and the official published version of record. People interested in the research are advised to contact the author for the final version of the publication, or visit the $\mathrm{DOI}$ to the publisher's website.

- The final author version and the galley proof are versions of the publication after peer review.

- The final published version features the final layout of the paper including the volume, issue and page numbers.

Link to publication

\section{General rights}

Copyright and moral rights for the publications made accessible in the public portal are retained by the authors and/or other copyright owners and it is a condition of accessing publications that users recognise and abide by the legal requirements associated with these rights.

- Users may download and print one copy of any publication from the public portal for the purpose of private study or research.

- You may not further distribute the material or use it for any profit-making activity or commercial gain

- You may freely distribute the URL identifying the publication in the public portal.

If the publication is distributed under the terms of Article 25fa of the Dutch Copyright Act, indicated by the "Taverne" license above, please follow below link for the End User Agreement:

www.tue.nl/taverne

Take down policy

If you believe that this document breaches copyright please contact us at:

openaccess@tue.nl

providing details and we will investigate your claim. 


\section{AD| $\begin{aligned} & \text { The Journal of } \\ & \text { Chemical Physics }\end{aligned}$}

\section{Effect of bending flexibility on the phase behavior and dynamics of rods}

Saber Naderi and Paul van der Schoot

Citation: The Journal of Chemical Physics 141, 124901 (2014); doi: 10.1063/1.4895730

View online: http://dx.doi.org/10.1063/1.4895730

View Table of Contents: http://scitation.aip.org/content/aip/journal/jcp/141/12?ver=pdfcov

Published by the AIP Publishing

\section{Articles you may be interested in}

The phase behavior, structure, and dynamics of rodlike mesogens with various flexibility using dissipative particle dynamics simulation

J. Chem. Phys. 133, 144911 (2010); 10.1063/1.3503602

Characteristic behavior of short-term dynamics in reorientation for Gay-Berne particles near the nematic-isotropic phase transition temperature

J. Chem. Phys. 125, 204902 (2006); 10.1063/1.2393238

Studies of translational diffusion in the smectic A phase of a Gay-Berne mesogen using molecular dynamics computer simulation

J. Chem. Phys. 120, 394 (2004); 10.1063/1.1630014

Simulation study of the phase behavior of a primitive model for thermotropic liquid crystals: Rodlike molecules with terminal dipoles and flexible tails

J. Chem. Phys. 112, 9092 (2000); 10.1063/1.481520

Computer simulation studies of anisotropic systems. XXX. The phase behavior and structure of a Gay-Berne mesogen

J. Chem. Phys. 110, 7087 (1999); 10.1063/1.478563

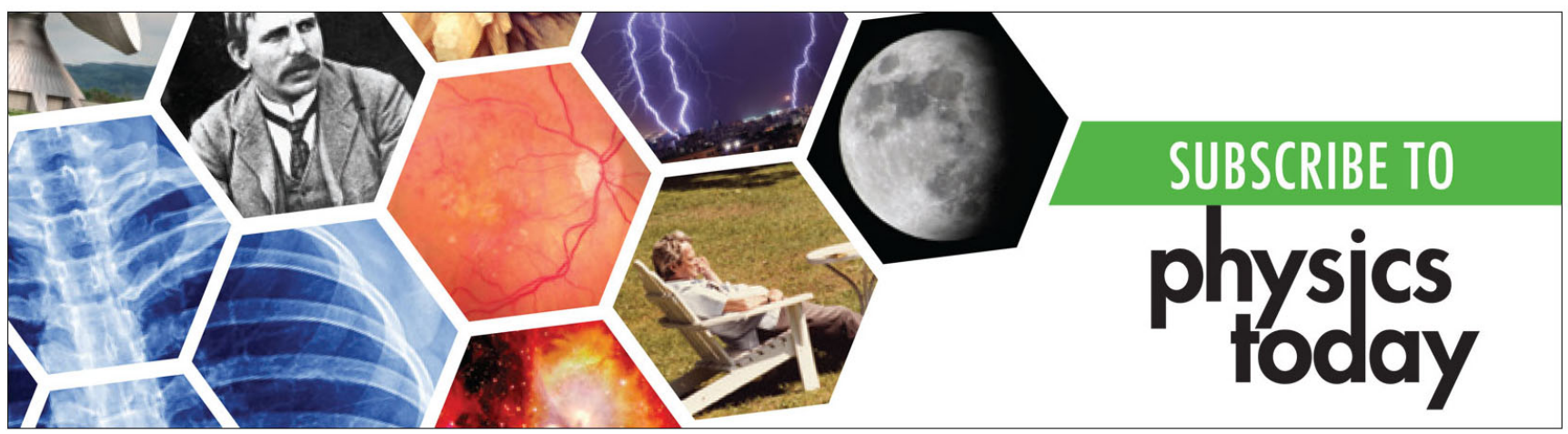




\title{
Effect of bending flexibility on the phase behavior and dynamics of rods
}

\author{
Saber Naderi ${ }^{1,2, a)}$ and Paul van der Schoot ${ }^{1,3}$ \\ ${ }^{1}$ Faculteit Technische Natuurkunde, Technische Universiteit Eindhoven, Postbus 513, \\ 5600 MB Eindhoven, The Netherlands \\ ${ }^{2}$ Dutch Polymer Institute, P.O. Box 902, 5600 AX Eindhoven, The Netherlands \\ ${ }^{3}$ Instituut voor Theoretische Fysica, Universiteit Utrecht, Leuvenlaan 4, 3584 CE Utrecht, The Netherlands
}

(Received 2 September 2014; accepted 3 September 2014; published online 22 September 2014)

\begin{abstract}
We study by means of molecular and Brownian dynamics simulations the influence of bending flexibility on the phase behavior and dynamics of monodisperse hard filamentous particles with an aspect ratio of 8 and persistence lengths equal to 3 and 11 times the particle length. Although our particles are much shorter, the latter corresponds to the values for wild-type and mutant $f d$ virus particles that have been subject of a recent experimental study, where the diffusion of these particles in the nematic and smectic-A phase was investigated by means of video fluorescence microscopy [E. Pouget, E. Grelet, and M. P. Lettinga, Phys. Rev. E 84, 041704 (2011)]. In agreement with theoretical predictions and simulations, we find that for the more flexible particles (shorter persistence length) the nematic $(\mathrm{N})$ to smectic-A (Sm-A) phase transition shifts to larger values of the particle density. Interestingly, we find that for the more rigid particles (larger persistence length), the smectic layer-tolayer distance decreases monotonically with increasing density, whereas for the more flexible ones, it first increases, reaches a maximum and then decreases. For our more flexible particles, we find a smectic-B phase at sufficiently high densities. Moreover, in line with experimental observations and theoretical predictions, we find heterogeneous dynamics in the Sm-A phase, in which particles hop between the smectic layers. We compare the diffusion of our two types of particle at identical values of smectic order parameter, and find that flexibility does not change the diffusive behavior of particles along the director yet significantly slows down the diffusion perpendicular to it. In our simulations, the ratio of diffusion constants along and perpendicular to the director decreases just beyond the N-Sm-A phase transition for both our stiff and more flexible particles. () 2014 AIP Publishing LLC. [http://dx.doi.org/10.1063/1.4895730]
\end{abstract}

\section{INTRODUCTION}

Liquid crystals are states of condensed matter with a level of ordering in between that of liquids, exhibiting shortrange positional order, and crystals that display long-range positional and rotational order. ${ }^{1}$ A host of liquid-crystalline phases have been found in dispersions of highly anisotropic colloidal particles, such as rod- and plate-like ones, phases that have long- or quasi long-range order in some directions while they exhibit short-range correlations in others. ${ }^{1}$ For example, dispersions of rod-like colloids, including $f d$ virus, TMV, and DNA, are known to form (chiral) nematic liquid crystals at sufficiently high concentrations, in which the particles spontaneously align their principal axes along a common axis known as the director. ${ }^{2-5}$ Onsager $^{6}$ explained this spontaneous alignment theoretically by showing that long rods that interact via excluded-volume interactions self-organize into a nematic phase by optimizing the sum of translational and rotational entropy. Simulations on long rod-like particles have since confirmed this for spherocylinders and ellipsoids of revolution. ${ }^{7-9}$

Computer simulations and density functional theory calculations have also shown that at sufficiently high densities a nematic-smectic-A phase transition occurs in dispersions

\footnotetext{
a)sbrnaderi@gmail.com
}

of monodisperse hard spherocylinders. ${ }^{7,8,10-13}$ Particles in the smectic-A phase form layers and exhibit quasi-long-range positional ordering along the director, while in the direction perpendicular to it they behave like a liquid. McGrother et al. ${ }^{11}$ found that for spherocylinders with relatively small length to diameter ratios, $L / D=3.2$, a transition directly from the isotropic to the smectic-A phase occurs. For larger aspect ratios, a nematic phase intervenes before the smectic-A phase appears at more elevated densities. Smectic ordering has been observed in solutions of monodisperse stiff rod-like particles, such as poly( $\gamma$-benzyl L-glutamate), silica rods, and $\mathrm{TMV},{ }^{14-16}$ as well as in solutions of semi-flexible filamentous ones, e.g., $f d$ virus. ${ }^{17}$

Although both rigid and semi-flexible filamentous particles can form a smectic-A phase, theoretical and simulation studies have shown that increasing the particle bending flexibility shifts the concentration at which the nematic to smectic phase transition occurs to higher values, ${ }^{18-21}$ while it also decreases the smectic layer spacing. ${ }^{18}$ The experimental observations of Dogic and Fraden, ${ }^{17}$ who studied the nematicsmectic phase transition in suspensions of semi-flexible $f d$ virus particles, confirmed this. Within a second-virial approximation, Hidalgo et $_{\text {al. }}{ }^{20}$ found from density functional theory calculation that for infinitely rigid rods the nematic-smectic phase transition must be of second order whereas for semiflexible particles the transition is a weakly first order one. 
Particle bending flexibility not only influences the nematic-smectic phase transition but it also changes the Brownian kinetics of individual particles in these phases. The study of kinetics at the level of an individual particle in suspensions of colloidal particles has been made possible by the recent advances in experimental techniques, such as fluorescence microscopy. ${ }^{22-25}$ Lettinga et al. ${ }^{25,26}$ measured the self-diffusion of semi-flexible $f d$ virus particles in isotropic and nematic phases. In qualitative agreement with simulations of hard spherocylinders ${ }^{27}$ and ellipsoids, ${ }^{28}$ they found that the ratio of the diffusion constant parallel to the director $\left(D_{\|}\right)$and the one perpendicular to it $\left(D_{\perp}\right)$ increases as the nematic order parameter increases. In agreement with experiments, $D_{\|}$initially increases and subsequently decreases upon reaching the nematic-smectic transition. ${ }^{27,28}$

The unusual self-diffusion of filamentous particles in the smectic-A phase has been the center of attention in computer simulations, theoretical studies and experiments. ${ }^{29-32}$ Lettinga et al. $^{29,30}$ studied the self-diffusion of individual particles in a suspension of semi-flexible $f d$ virus at concentrations for which a smectic-A phase is stable. The authors observed a hopping-type diffusion, in which $f d$ particles mostly rattle around their equilibrium positions in a smectic layer and occasionally jump from one layer to another. Later, it was shown in a study based on dynamical density functional theory that this hopping-type diffusion is dictated by a temporary caging of particles by their direct neighbors that competes with the permanent self-consistent molecular field induced by all other particles. ${ }^{30,31}$ A similar type of inter-layer diffusion was observed in the smectic phases of monodisperse rigid rod-like silica particles and in this case, in contrast to the above mentioned experiments involving semiflexible particles, layer-to-layer diffusion was slower than the in-layer diffusion. ${ }^{32}$ This indicates that particle bending flexibility may enhance the inter-layer diffusion and/or it may slow down the in-layer diffusion. In order to shed light on this we here report on molecular dynamics and Brownian dynamics (BD) simulations in which we probe the influence of bending flexibility on the self-diffusion of filamentous particles on both sides of the nematic-Smectic-A phase transition.

Before presenting our own simulations it is of interest to mention that simulation studies on the diffusion of parallel and of freely rotating hard spherocylinders in the smectic phase have confirmed that diffusion along the director is indeed of the hopping-type, while that perpendicular to it is typical of a dense fluid with a relatively fast relaxation dynamics. ${ }^{33-35}$ Cinacchi and De Gaetani ${ }^{36}$ investigated the mechanism of diffusion of stiff wormlike particles in the smectic-A phase by molecular dynamics simulations. For sufficiently long timescales, where the mean square displacement parallel and perpendicular to the director exhibits a diffusive behavior, the value of $D_{\|}$was found to be smaller than $D_{\perp}$. This is in agreement with experimental data on the diffusion of silica rods in the smectic-A phase, ${ }^{32}$ but contrasts with findings on the diffusion of semi-flexible $f d$ virus in the same phase. $^{29,30}$ The difference again might be due to the flexibility of the $f d$ virus, or alternatively it might be caused by the fact that silica rods in the experiments of Kuijk et al. ${ }^{32}$ and worm- like particles in the simulations of Cinacchi and De Gaetani ${ }^{36}$ have much smaller aspect ratios than $f d$ virus.

Pouget et al. ${ }^{37,38}$ performed experiments on aqueous dispersions of wild-type $f d$ virus $(f d-w t)$ and a stiffer mutant $(f d$ $Y 21 M$ ). The length, $L$, of both variants of $f d$ virus is identical, approximately $880 \mathrm{~nm}$, but their persistence lengths, $L_{p}$, differ. Of the former, $L_{p}=2800 \pm 700 \mathrm{~nm}$, while that of the latter, $L_{p}=9900 \pm 1600 \mathrm{~nm}$, giving for the ratio $L / L_{p}$ values of 0.31 and $0.09 .{ }^{37}$ The authors found that for both $f d$-wt and $f d-Y 21 M$ in nematic and in smectic phases the ratio $D_{\|} / D_{\perp}$ is much larger than unity, showing that it is not due to particle bending flexibility that in the smectic-A phase of $f d$ virus $D_{\|}$is larger than $D_{\perp}$. Interestingly, for more rigid virus particles the value of $D_{\|} / D_{\perp}$ decreases with increasing density in the smectic phase whereas it increases for the case of $f d$ $w t$ particles. The latter happens because for the more flexible particles $D_{\perp}$ decreases more strongly with increasing concentration than $D_{\|}$does.

To get a more detailed insight in the influence of bending flexibility on the dynamics of particles in the nematic and the smectic-A phase, we embark upon a Brownian dynamics simulation study of rod-like particles. Our fused-sphere representation of the rods have an aspect ratio of 8.0 and length-overpersistence-length ratios of $L / L_{p}=0.09$ and 0.31 , mimicking the flexibilities of $f d-w t$ and $f d-Y 21 M$. With current computer power it is not quite feasible to also get the same aspect ratio as that of the virus particles. First, we carry out simulations in isobaric-isothermal ensemble in order to obtain the phase diagram of our particles that interact via a soft repulsive potential. Next, we run Brownian dynamics simulations and study the diffusion of single particles in the nematic and smectic-A phases. We find that by entering the smectic phase, the ratio of $D_{\|} / D_{\perp}$ decreases for both values of $L / L_{p}$ and becomes less than unity for the case of the more rigid particles with $L / L_{p}=0.09$.

The remainder of this paper is organized as follows. In Sec. II, we describe our simulation model and the way we analyze our simulation data. Equilibrium properties and phase behavior of our filamentous particles at different values of pressure are discussed in Sec. III. In Sec. IV, we present our simulation results on the kinetics of the filamentous particles on both sides of the N-Sm-A transition. A summary of our work is given in Sec. V.

\section{MODEL AND SIMULATION METHODS}

We perform simulations using LAMMPS molecular dynamics package ${ }^{39}$ with $\mathrm{N}=4464$ filamentous particles in the simulation box. Each of the particles is modeled as a chain made up of $n=17$ spherical beads. Within a chain, adjacent beads are connected to each other via a harmonic bond potential of the form $U_{b o n d}(r)=k_{b}\left(r-l_{b}\right)^{2}$, where $r$ is the distance between the two beads, $l_{b}=0.5 \sigma$ is the equilibrium bond length with $\sigma$ being the bead diameter, and $k_{b}$ is the strength of the potential. To ensure a fixed bond length in our simulation, we choose a large value for the strength of bond potential, $k_{b}=50 k_{B} T / \sigma^{2}$, where $k_{B} T$ is the thermal energy with $k_{B}$ Boltzmann's constant and $T$ the absolute temperature. Each three bonded beads are in addition connected via a harmonic 
bending potential of the form $U_{\text {bend }}(\theta)=k_{a}(\theta-\pi)^{2}$, where $\theta$ is the angle that is formed by the two bonds that link these three beads together and $k_{a}$ is the strength of the bending potential that determines rigidity of a filamentous particle. In the limit where $n \rightarrow \infty$ and $k_{b} l_{b}^{2} / k_{B} T \gg 1, k_{a}$ can be linked to the persistence length of a particle via a simple relation, $L_{p}=2 k_{a} l_{b} / k_{B} T$. $^{40,41}$

We carry out our simulations with two values of contour length over persistence length ratio $L / L_{p}=0.09$ and 0.31 , where $L=(n-1) l_{b}$. The total length of a particle is $(n-1) l_{b}+\sigma$, which in our simulations equals $9.0 \sigma$. All beads, excluding those that are nearest- or next-nearestneighbors in a chain, interact with each other via the repulsive part of the shifted Lennard-Jones potential,

$$
U_{L J}(r)= \begin{cases}4 \epsilon\left(\left(\frac{\sigma}{r}\right)^{12}-\left(\frac{\sigma}{r}\right)^{6}+\frac{1}{4}\right) & \text { if } r \leq 2^{\frac{1}{6}} \sigma, \\ 0 & \text { if } r>2^{\frac{1}{6}} \sigma,\end{cases}
$$

where $\epsilon$ is the strength of the interaction potential, which in our simulations is equal to the thermal energy, $k_{B} T$, and $r$ is again the distance between the centers of mass of the beads. We choose this potential to mimic the soft screened electrostatic repulsion between the charge-stabilized $f d$ virus particles in the experiments of Grelet and collaborators. ${ }^{42,43}$

To obtain the phase diagram of our particles, we run simulations in the isobaric-isothermal (NPT) ensemble at different pressures, starting from an AAA crystal initial configuration. We incrementally expand the system from the highest pressure, $P^{*}=5.0$, to the lowest one, $P^{*}=1.0$, where $P^{*}$ is related to the actual pressure of the system, $P$, by $P^{*}=P \sigma^{3} / k_{B} T$. In each step, we first slowly decrease the pressure in a short simulation run of $10^{5}$ simulation steps and after that we carry out a $N P T$ simulation at the final value of pressure with $6 \times 10^{6}$ time steps. To control the temperature and pressure in our simulations, we make use of Nose-Hoover thermostat and barostat. See Sec. III for a discussion on the shape of our simulation box. To check for a potential effect of hysteresis in our simulations, we compress the system again after reaching the nematic phase at the lowest pressure tested. In all our NPT simulations, the linear dimensions of our simulation box can change independently, which allows the system to relax properly without unphysical effects due to the finite size of our system.

To study the dynamics of our particles in the nematic and smectic phases, we carry out BD simulations. Hence, we ignore hydrodynamic interactions that might be important. ${ }^{26}$ We use the final state of a relaxed system obtained from a $N P T$ molecular dynamics simulation at a given pressure as the initial state for a BD simulation at a particle density corresponding to that pressure. This way we make certain that the systems are relaxed at the given densities. Depending on particle density in the BD simulations, we choose a time step between $10^{-3}$ and $5 \times 10^{-3} t^{*}$ where $t^{*}$ is the unit of time, set by the self-diffusion constant of a single bead $D_{b}=\sigma^{2} / t^{*}$ and we run simulations for a total of $6 \times 10^{6}$ time steps. Our particles are made up of 17 beads, so in the free-draining limit of our simulations their self-diffusion constant is $D=D_{b} / 17 .^{44}$ In our simulations, the self-diffusivities of the elongated particles along and perpendicular to their long axis are equal in the dilute (non-interacting) limit. We ignore the fact that these dilute-limit self-diffusion constants differ by a factor of two, to keep the computational complexity of our simulation code at a reasonable level. ${ }^{44}$

The equilibrium properties and the dynamics of our system at different pressures and densities, we probe by computing (i) the pair-correlation function, $g$, (ii) the nematic order parameter, $S_{2}$, (iii) the smectic order parameter, $\tau_{s}$, (iv) freeenergy barriers between layers of the smectic phase, (v) the bond orientational order parameter, $\Psi_{6}$, (vi) the mean-square displacement of particles, and (vii) the self part of the Van Hove correlation function, $G_{s}$. The pair-correlation function is calculated for the directions parallel and perpendicular to the nematic director. The former is defined as

$$
g_{\|}(r)=\frac{1}{N}\left\langle\frac{1}{\rho} \sum_{i} \sum_{j \neq i} \delta\left[r-\mathbf{r}_{i j} \cdot \hat{\mathbf{n}}\right]\right\rangle,
$$

where $\delta$ is the Dirac delta function, $\mathbf{r}_{i j}$ is the distance between the beads in the middle of the ith and the jth filamentous particle, $\hat{\mathbf{n}}$ is the nematic director, $\rho=N / V$ is the particle density with $V$ being the volume of the simulation box, and the angular brackets denote an ensemble average. The pair-correlation function perpendicular to the director is defined as

$g_{\perp}(r)=\frac{1}{N}\left\langle\frac{1}{\rho} \sum_{i} \sum_{j \neq i} \delta\left[r-\left|\mathbf{r}_{i j} \times \hat{\mathbf{n}}\right|\right] \Theta\left(\frac{L}{2}-\mathbf{r}_{i j} \cdot \hat{\mathbf{n}}\right)\right\rangle$,

where $\Theta$ is the Heaviside function and $L$ is the length of a particle.

The nematic order parameter is a measure of the degree of orientational ordering of the particles. To calculate it, we first compute for each snapshot of our simulations the orientational order tensor with components given by

$$
Q_{\alpha \beta}=\frac{1}{N} \sum_{i}^{N}\left(\frac{3}{2} \hat{e}_{i \alpha} \hat{e}_{i \beta}-\frac{1}{2} \delta_{\alpha \beta}\right),
$$

where $\alpha$ and $\beta$ are $x, y, z$ directions, $\hat{e}_{i}$ is a unit vector along the main body axis of a particle, which is defined along the line that connects the first bead to the last bead of the particle, and $\delta$ is the Kronecker delta. To obtain the nematic order parameter, $S_{2}$, we calculate the eigenvalues and eigenvectors of this tensor. The largest eigenvalue is the nematic order parameter and the eigenvector associated with it is the nematic director. We compute the nematic order parameter and director by averaging over the nematic order parameters that are calculated for each simulation snapshot in simulation run, so we are time-averaging the order parameter. In the course of our simulation, we find the director not to fluctuate much.

We also compute the smectic order parameter, which is a measure of positional ordering in the direction of the nematic director. It can be calculated by maximizing the following relation with respect to $d$ :

$$
f(d)=\left|\frac{1}{N} \sum_{j=1}^{N} \exp \left(i 2 \pi \frac{\mathbf{r}_{\mathbf{j}} \cdot \hat{\mathbf{n}}}{d}\right)\right|,
$$

where $\mathbf{r}_{\mathbf{j}}$ is the position of the jth particle and the smectic order parameter is defined $\tau_{s}=\max f(d)$. The smectic order 
parameter for each simulation is calculated by averaging over those computed for each simulation snapshot. In the smectic phase, the value of $d$ that maximizes $f(d)$ is the smectic layer-to-layer distance that we refer to as $d_{s m}$. In this phase, there is a self-consistent molecular field that organizes particles in the smectic layers and therefore there is a free-energy barrier between the equilibrium layer positions. By calculating the probability of finding a particle at a position $z$ along the nematic director, $\Pi(z)$, we compute the free-energy barrier by using the following relation, $\beta U(z)=-\ln \Pi(z)$, where $\beta=1 / k_{B} T$ with $k_{B}$ the Boltzmann factor and $T$ the temperature and $\ln$ denotes the natural logarithm. ${ }^{29}$

To obtain a measure for the level of ordering within the smectic layers, we calculate the bond orientational order parameter, $\Psi_{6}$, which is given by

$$
\begin{aligned}
\psi_{6}= & \frac{1}{3 N} \mid \sum_{i} \sum_{j} \exp \left(6 i \theta_{i j}\right) \Theta\left(r_{p}-\mathbf{r}_{i j} \cdot \hat{\mathbf{n}}\right) \\
& \times \Theta\left(r_{l}-\left|\mathbf{r}_{i j} \times \hat{\mathbf{n}}\right|\right) \mid,
\end{aligned}
$$

where $\theta_{i j}$ is the angle between the projection of $\mathbf{r}_{i j}$ on the plane perpendicular to $\hat{\mathbf{n}}$ and a fixed axis in this plane, $r_{p}=L / 2$ and $r_{l}=1.35 \sigma$ are chosen in such a way that only for the nearest-neighbors the product of the two Heaviside functions is non-zero. Again, for each simulation we calculate $\psi_{6}$ order parameter for each snapshot and take its average over all snapshots.

Diffusion of particles in the nematic and smectic phase, we investigate by computing the mean-square displacement along the director, $\left\langle(\Delta \mathbf{r}(t) \cdot \hat{\mathbf{n}})^{2}\right\rangle$, and that perpendicular to it, $\left\langle|\Delta \mathbf{r}(t) \times \hat{\mathbf{n}}|^{2}\right\rangle$. Note that in our simulations $\hat{\mathbf{n}}$ does not change significantly over time (results not shown). In addition, we calculate the self part of the van Hove function, which is a measure of the probability of finding a particle at a given distance from its initial position, after a time interval of $t$. For the direction along the director, it can be defined as

$$
G_{s}^{\|}(z, t)=\frac{1}{N}\left\langle\sum_{i=1}^{N} \delta\left[z+z_{i}\left(t_{0}\right)-z_{i}\left(t+t_{0}\right)\right]\right\rangle,
$$

where $z_{i}(t)=\mathbf{r}_{i}(t) \cdot \hat{\mathbf{n}}$ and for the direction perpendicular to the director, $G_{s}^{\perp}$, is given by

$$
G_{s}^{\perp}(R, t)=\frac{1}{2 \pi N R}\left\langle\sum_{i=1}^{N} \delta\left[R+R_{i}\left(t_{0}\right)-R_{i}\left(t+t_{0}\right)\right]\right\rangle,
$$

where $R_{i}(t)=\left|\mathbf{r}_{i}(t) \times \hat{\mathbf{n}}\right|$.

\section{PHASE BEHAVIOR OF THE FILAMENTOUS PARTICLES}

As mentioned above, we perform MD simulations in an isobaric-isothermal ensemble in order to obtain the phase diagram of our particles. To this end, we incrementally expand our simulation box starting from an AAA crystal in which $N=4464$ filamentous particles are arranged in 16 layers along the $z$ axis of the simulation box. We choose this type
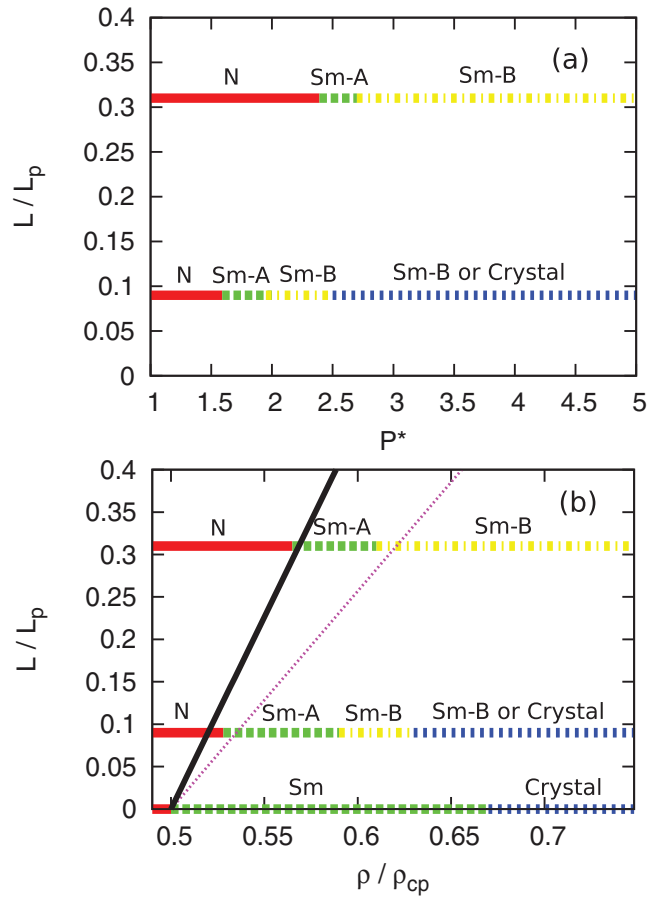

FIG. 1. Phase diagram of our filamentous particles for three values of the length-over-persistence-length ratios $L / L_{p}=0.31,0.09$, and 0 as a function of (a) reduced pressure, $P^{*}$ and (b) reduced volume density $\rho / \rho_{c p}$ where $\rho_{c p}$ is the closed packed density for our particles. The red solid line and the green dashed line indicate the range in which the nematic and smectic-A phases are stable, respectively. The blue dotted line shows the region where the smectic$\mathrm{B}$ or crystalline phases are stable and the yellow dotted-dashed line indicates the range in which the smectic-B phase is stable. The black solid line and purple dotted line correspond to theoretical predictions for nematic-smecticA transition for $\beta=0.0235$ and $S_{2} \approx 0.84$ and 0.9 , respectively. See the main text.

of elongated box to avoid unphysical correlations in the $z$ direction, which is initially along the long axis of all particles. For both types of particles with $L / L_{p}=0.31$ and 0.09 , we do expansion simulations in which we decrease the dimensionless pressure from $P^{*}=5.0$ to $P^{*}=1.0$. Next, we compress our simulation box by increasing pressure starting from the final snapshot of our simulations at the lowest value of the pressure in our expansion simulations.

Before proceeding with a detailed analysis of our simulations, in the next two paragraphs we first present in Fig. 1(a) the phase diagram resulting from our calculations for the two values of $L / L_{p}$ as a function of reduced pressure. As can be seen in this figure, the N-Sm-A phase transition occurs at a larger value of the pressure for the more flexible particles with $L / L_{p}=0.31$. The region in which the Sm-A phase is stable is also smaller for these particles. This is because at pressures larger than $P^{*} \approx 2.6$, the more flexible particles self-organize into a smectic-B phase in which the rods exhibit hexagonal ordering within the smectic layers. As we shall discuss later, in the smectic-B phase that these particles form the layers are randomly displaced with respect to each other, which causes the bond-orientational order parameter, $\psi_{6}$, to be approximately zero if averaged over all the layers. Our results are supported by very recent experiments on the phase behavior of fd virus particles in which for flexible particles 
a smectic-B phase has been observed between the smectic-A and columnar phases. ${ }^{45}$

In contrast, for the more rigid particles, we also observe the smectic-B phase in a region between $P^{*} \approx 2.0$ and 2.5 . However, increasing pressure to values larger than $P^{*} \approx 2.5$ results in arrangement of particles in layers with hexagonal ordering, which are not displaced randomly. Consequently, the value of $\psi_{6}$ is larger than zero in this case and therefore, because we cannot measure the long-range hexagonal order in our system due to the finite size of our simulation box, we conclude that the phase formed by the particles can be either a smectic-B phase or a crystalline one.

In Fig. 1(b), the phase diagram is presented for our two values of $L / L_{p}$ as well as for rigid spherocylinders, i.e., $L / L_{p}$ $=0$, as a function of reduced density, $\rho / \rho_{c p}$, where $\rho_{c p}$ is the closed-pack density of our particles. The results corresponding to rigid spherocylinders we obtained from the simulation results of Bolhuis and Frenkel ${ }^{8}$ for rods with the same aspect ratio as that of our particles. The black and purple lines are theoretical predictions for the nematic-smectic-A phase transition from the work of van der Schoot. ${ }^{18}$ According to this theory, the nematic-smectic spinodal line is given by $\rho=\rho_{r}\left(1+\beta L \alpha_{0} / L_{p}\right)$, where $\rho_{r}$ is the nematic-smectic-A transition density for rigid rods, $\beta$ is a constant calculated in the work of van der $\operatorname{Schoot}^{18}$ and $\alpha_{0} \approx 3 /\left(1-S_{2}\right)$ is related to the nematic order parameter, $S_{2}$, of the rigid rods at $\rho=\rho_{r}$.

The black and purple lines in Fig. 1(b) are obtained from the above mentioned formula with $\rho_{r}=0.50, \beta=0.0235$, and $S_{2} \approx 0.84$ and 0.9 , respectively. The value of $S_{2}=0.84$ corresponds to the nematic ordering of our particles with $L / L_{p}$ $=0.09$ at the nematic-smectic transition and gives a quantitative agreement with our simulation data. $S_{2} \approx 0.9$ is the nematic order parameter that is equivalent to $\alpha_{0}=33$ computed by Bladon and Frenkel ${ }^{19}$ from the orientational distribution of rigid rods with an aspect ratio of 6 at $\rho_{r}=0.51$. For this value agreement is not as good but still semi-quantitative. In the remaining of this section, we focus attention on an in-detail analysis of the simulation data and measurement of nematic, smectic, and bond orientational order parameters.

To measure the level of orientational ordering in our simulations, we compute the nematic order parameter, $S_{2}$, as indicated in Sec. II. Shown in Fig. 2 is $S_{2}$ as a function of $P^{*}$ for the two values of $L / L_{p}$ and for both our expansion and compression simulations. For all values of the pressure $S_{2} \rightarrow 1$, which shows that particles are almost perfectly parallel in our simulations. At high pressures, the values of the nematic order parameter obtained from our expansion simulations are lower than those from the compression simulations. The discrepancy is presumably caused by the fact that we start from an AAA crystal structure, which is not necessarily the equilibrium crystalline structure for our particles at that pressure. ${ }^{8}$ By looking at the snapshots of our expansion simulations at high pressures, we find that the particles in the layers are slightly tilted with respect to each other (reminiscent of the smectic-C phase), which results in a smaller value for the nematic order parameter. This is also why there is a jump in the value of $S_{2}$ at $P^{*}=2.7$ (for $L / L_{p}=0.31$ ) and $P^{*}=2.0$ (for $L / L_{p}=0.09$ ) as the pressure decreases. The jump results from the relaxation of layers at lower pres-

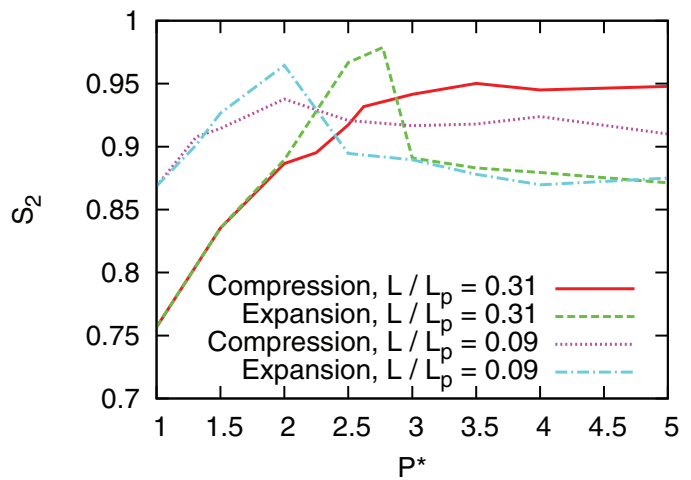

FIG. 2. Nematic order parameter, $S_{2}$, as a function of reduced pressure $P^{*}$ for $N P T$ simulations in which we vary the pressure of the system starting from $P^{*}=5.0$ in an AAA crystalline state (expansion simulations) and for simulations where we increase the pressure from $P^{*}=1.0$, starting from the final state of a system in expansion simulations at $P^{*}=1.0$ (compression simulations). Both expansion and compression simulations are performed with two types of particles with different flexibilities and length, $L$, over persistence length, $L_{p}$, of $L / L_{p}=0.31$ and 0.09 .

sures and elimination of the tilt that is observed at higher pressures.

The reason why we started from an AAA crystalline structure is that we initially aimed at investigating whether the internal flexibility of particles can force them to self-organize into a columnar phase rather than a smectic-A phase. In agreement with the simulations of Veerman and Frenkel, ${ }^{46}$ we find that for sufficiently small systems a meta-stable columnar phase does indeed form (results are not shown), but for the large systems that we study here no stable columnar phase presented itself.

As discussed above, the value of $S_{2}$ is close to unity for all the values of pressure between $P^{*}=1.0$ and 5.0. In order to find out what is the highest pressure at which the nematic phase is stable, we calculate the smectic order parameter, $\tau_{s}$, as described above. Shown in Fig. 3 is $\tau_{s}$ as a function of the dimensionless pressure $P^{*}$ and the dimensionless density $\rho / \rho_{c p}$ for the two values of $L / L_{p}=0.31$ and 0.09 , where we first expand our system from $P^{*}=5$ to 1 and then cycle back to a value of 5. Here, $\rho$ is the average number density of particles at a given pressure and $\rho_{c p}$ is the close packing density of spherocylinders, which is given by $\rho_{c p}=2 /(\sqrt{2}+(L / D) \sqrt{3}) D^{3}$, where $L / D=8$ is the aspect ratio of our particles. For the case of the more flexible particles with $L / L_{p}=0.31$, the N-Sm-A phase transition occurs at higher values of pressure and density. This is in agreement with theoretical predictions, ${ }^{18,21}$ simulations, ${ }^{19}$ and experiments. ${ }^{17}$ From the behavior of the smectic order parameter as a function of pressure, one could argue that the $\mathrm{N}-\mathrm{Sm}-\mathrm{A}$ transition for our particles is of the second order. However, we also observe that there is hysteresis in our simulations: the dependence of the particle density as a function of the pressure in the compression part of our simulations differs slightly from that in the expansion part (data not shown) as does the smectic order parameter shown in Fig. 3, which is an indication that the transition must be of the first order. Therefore, from our simulation results we cannot determine the order of this transition. 

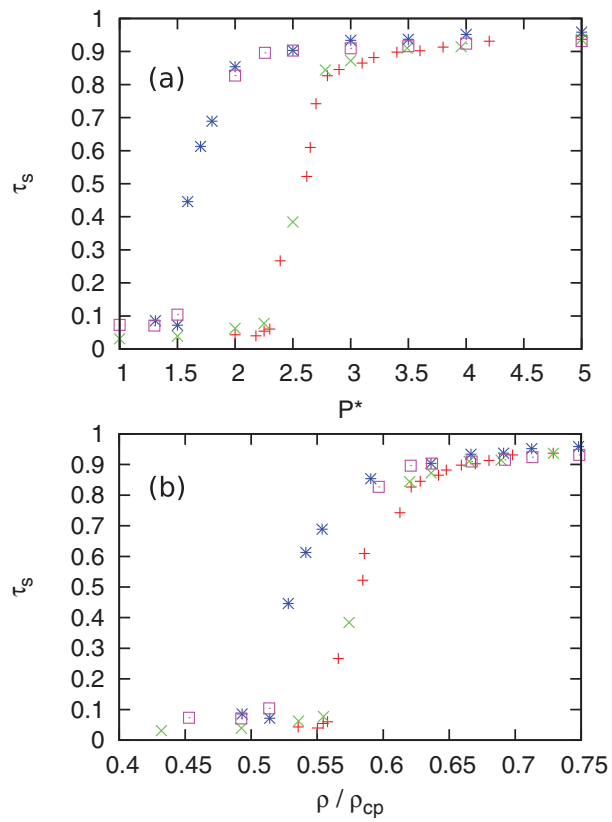

FIG. 3. Smectic order parameter, $\tau_{s}$, as a function of the reduced pressure $P^{*}$ (a) and particle density, $\rho$ (b). Here, $\rho_{c p}$ is the close packing density of spherocylinders (see the main text). Purple squares and blue stars correspond to initial expansion and subsequent compression simulations of our filamentous particles with $L / L_{p}=0.09$. Red pluses and green crosses are associated with compression and expansion simulations with filamentous particles with $L / L_{p}=0.31$.

The smectic layer-to-layer distance, $d_{s m}$, is another quantity of interest. Shown in Fig. 4 is $d_{s m}$ as a function of smectic order parameter, $\tau_{s}$, for the two values of $L / L_{p}$ investigated. For the more flexible particles, $d_{s m}$ has a smaller value any given $\tau_{s}$. This is because the effective (projected) length of these particles is smaller than that of more rigid particles due to particle flexing. Therefore, these particles form shorter layers especially at relatively low densities at which there is more space for undulations within the smectic layers. As the density increases, the particle-particle spacing within the lay-

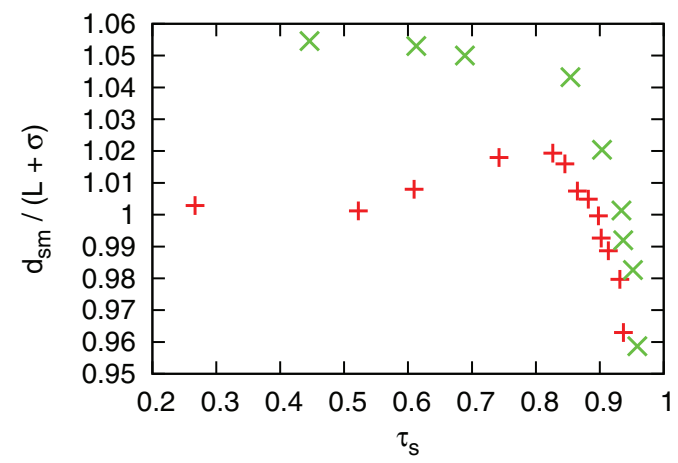

FIG. 4. The smectic layer-to-layer distance, $d_{s m}$, in the smectic phase as a function of smectic order parameter $\tau_{s}$, obtained from compression simulations for $L / L_{p}=0.31$ (red pluses) and $L / L_{p}=0.09$ (green crosses). Here, $L_{p}, L$, and $L+\sigma$ are the persistence length, the contour length, and the total length of an isolated particle, respectively, with $\sigma$ being the diameter of our beads. Because the particles compress slightly at high pressures corresponding to large values of the smectic order parameter $d_{s m} /(L+\sigma)$ can be smaller than unity even though in reality $d_{s m}$ is in that case virtually equal to the actual rod length. ers becomes smaller and the effective length of particles increases, which leads to an increase in the value of $d_{s m}$. In our simulations, for the more flexible particles $d_{s m}$ initially increases with increasing $\tau_{s}$ and after reaching a maximum value it decreases again, whereas for the case of more rigid particles it decreases monotonically. We note that $d_{s m}$ becomes slightly smaller than the optimal length of an isolated, $L$, at high pressures for both values of $L / L_{p}$. This is because our particles are made up of beads that are connected via harmonic bonds and, although the strength of the harmonic bonds is large $\left(k_{b}=50 k_{B} T / \sigma^{2}\right)$, particles at high pressures compress along their principal axis and form slightly thinner layers.

Particle flexing and undulation can also influence the level of ordering of particles within the smectic layers. To investigate this, we compute the bond orientational order parameter, $\psi_{6}$, which is a measure of hexagonal ordering in the direction perpendicular to the director. For a perfect hexagonal lattice, $\psi_{6}=1$, and for a system with no hexagonal order, $\psi_{6} \approx 0$. The bond orientational order parameter for the two values of $L / L_{p}$ are shown in Fig. 5 for both our expansion and compression simulations. The results of our expansion simulations show that the value of $\psi_{6}$ is smaller for the more flexible particles with $L / L_{p}=0.31$ than those for which $L / L_{p}$ $=0.09$. Moreover, in our expansion simulations, the value of $\psi_{6}$ for more rigid particles vanishes at lower values of the pressure (and density) compared to that of the more flexible ones showing that particle bending flexibility reduces the level of hexagonal ordering. Under recompression of the more rigid particles, we observe that $\psi_{6}$ attains a lower value at a given pressure compared to that of the expansion simulations, which is probably due to the fact that in the expansion simulations we start from a lattice with perfect hexagonal ordering. As we alluded to above, this is not a stable configuration even at the highest pressure tested. Here, our finding of hysteresis is also an indication that the smectic-A to smectic-B phase transition must be of first order. Our simulations show that for $P^{*}>2.5$ corresponding to densities $\rho / \rho_{c p}>0.59$ the more rigid particles self-organize into a phase where both $\psi_{6}$ and $\tau_{s}$ are non-zero. This phase may be a crystalline phase with

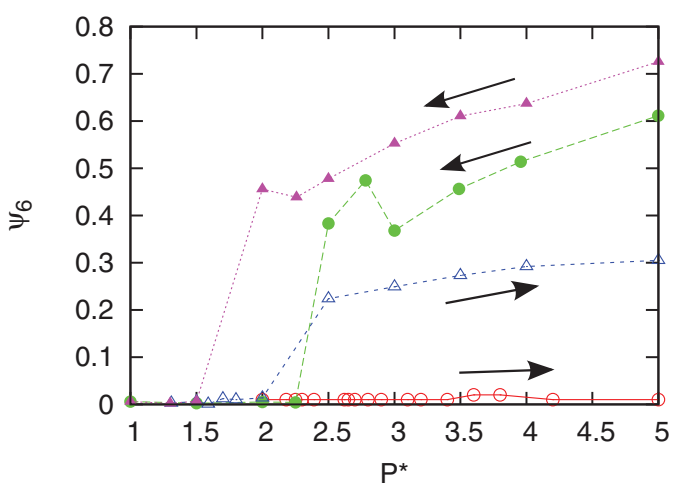

FIG. 5. The bond orientational order parameter, $\psi_{6}$, as a function of the reduced pressure, $P^{*}$. Blue open and purple filled triangles correspond to compression and expansion simulations of filamentous particles with $L / L_{p}$ $=0.09$. Red open and green filled circles are associated with compression and expansion simulations of filamentous particles with $L / L_{p}=0.31$. 


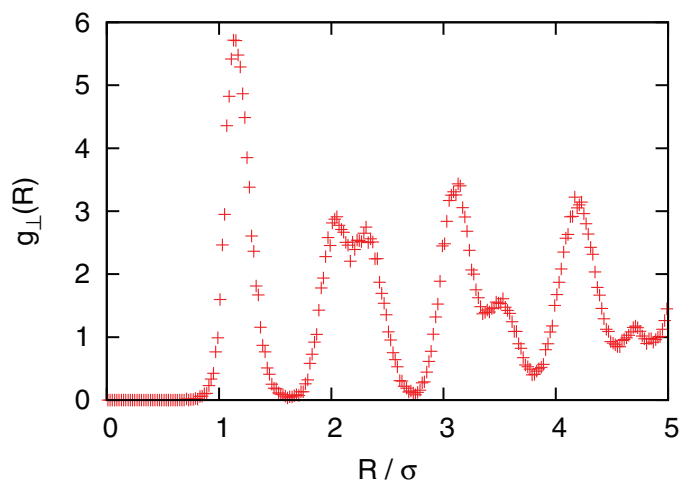

FIG. 6. The in-layer pair correlation function, $g_{\perp}$, as a function of the dimensionless transverse distance, $R / \sigma$, at a dimensionless pressure $P^{*}=5.0$ for particles with $L / L_{p}=0.31$. Results after expanding from $P^{*}=5.0$ to 1.0 and re-compression to $P^{*}=5.0$. Here, $\sigma$ is the diameter of beads that make up a particle.

long-range positional order or a smectic-B phase. Due to finite size of our simulation box we are not able to distinguish between these two phases.

Surprisingly, when we compress the nematic phase of the more flexible particles, the value of the $\psi_{6}$ order parameter is always very small even at the highest pressure, $P^{*}=5.0$, suggesting a smectic-A rather than a crystalline phase. To further investigate this, we calculate the pair correlation function within the smectic layers, $g_{\perp}(r)$. Shown in Fig. 6 is $g_{\perp}$ that is measured for the compression simulation at $P^{*}=5.0$. The first peak in $g_{\perp}$ appears at $R_{1} \approx 1.15$ and the second and third peaks appear at $2 R_{1}$ and $\sqrt{3} R_{1}$, respectively, showing that within the smectic layers the particles exhibit hexagonal ordering. This is characteristic of the smecticB phase that has also been found in other similar studies by Cinacchi and De Gaetani ${ }^{47}$ on shorter (semi-)flexible filamentous particles, although $\psi_{6}$ was larger than zero for what the authors call the "crystal (smectic-B) phase." Here, we find that particle bending flexibility favors the smectic-B phase against the crystal phase, which is in agreement with very recent experiments on wild-type $f d$ virus and its more rigid mutant. ${ }^{48}$

The reason why we do not see the hexagonal ordering in the $\psi_{6}$ order parameter is because the layers of hexagonally ordered particles are randomly displaced with respect to each other and therefore the contributions of layers to $\exp \left(6 i \theta_{i j}\right)$ in Eq. (6) cancel each. To pinpoint for what pressure the hexagonal ordering starts to increase, we calculate the $\psi_{6}$ order parameter for each layer of the smectic phase separately and after that compute its average over all layers. The results are shown in Fig. 7. As shown in this figure, the transition from smectic-A to smectic-B phase occurs at a pressure of approximately $P^{*}=2.7$ with an averaged in-layer order parameter $\psi_{6}$ of 0.28 . By comparing the values of averaged in-layer $\psi_{6}$ in Fig. 7 and $\tau_{s}$ in Fig. 3 we find that for the case of particles with $L / L_{p}=0.31$, the smectic-A phase is stable approximately between $P^{*}=2.4$ and $P^{*}=2.7$ corresponding to average densities between $\rho / \rho_{c p}=0.57$ and $\rho / \rho_{c p}=0.61$.

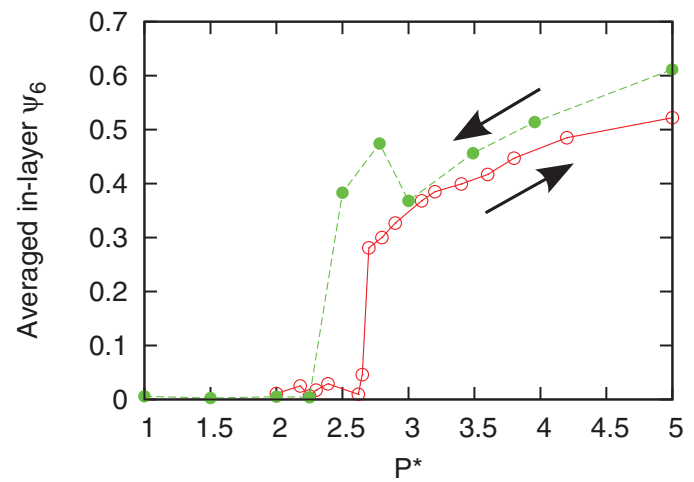

FIG. 7. The in-layer bond orientational order parameter, $\psi_{6}$, averaged over all the smectic layers in the system as a function of the reduced pressure, $P^{*}$, for particles with $L / L_{p}=0.31$ obtained from compression simulations (red open circles) and $\psi_{6}$ for particles with $L / L_{p}=0.31$ obtained from expansion simulations (green filled circles).

\section{DYNAMICS ON BOTH SIDES OF N-Sm-A PHASE TRANSITION}

As alluded to in Sec. I, recent experiments on wild-type and mutant $f d$ virus show that the ratio of diffusion constants of the particles parallel, $D_{\|}$, and perpendicular, $D_{\perp}$, to the director increases with increasing density for the case of more flexible wild-type $f d$ from the nematic phase entering the smectic-A phase whereas it decreases for the more rigid mutant. Inspired by this, we rely on Brownian dynamics simulations starting from the last configurations obtained from our compression MD simulations presented in Sec. III in order to study the kinetics of particles on both sides of the N-Sm-A phase transition.

In the smectic phase, long-time diffusion of particles along the director is dictated by the free-energy barriers resulted from the periodic self-consistent molecular field in this direction. ${ }^{31}$ To see how the free-energy barrier varies as a function of density, we calculate this quantity using the method that we described in Sec. II. The free-energy barrier, $U(z)$, is shown in Fig. 8 at four values of the reduced pressure, $P^{*}$, and for the two particle bending flexibilities corresponding to $L / L_{p}=0.31$ and 0.09 . For both types of particle, $U(z) \approx 0$ at the lowest value of the pressure that corresponds to a nematic phase. As expected, in the nematic phase particles do not feel a periodic self-consistent field along the director. In the smectic phase, the barrier height increases with increasing pressure (or density). The height of the barrier in the smectic-A phase ranges from $0.7 k_{B} T$ to $2.7 k_{B} T$ for the more flexible particles and from $2.1 k_{B} T$ to $4.0 k_{B} T$ for the more rigid ones. The barrier heights obtained from the smectic phase of $f d$ virus range between $0.66 k_{B} T$ and $4 k_{B} T .^{29,38}$

The presence of potential barriers along the director in the smectic-A phase leads to a heterogeneous kind of dynamics in this direction discussed in the Introduction. Particles in the smectic phase mostly rattle around their equilibrium positions in layers and from time to time they overcome this potential barrier and hop from one layer to another. The concomitant heterogeneous dynamics that is the result of this can be quantified by considering the self part of the Van Hove function along the director, $G_{s}^{\|}$. Shown in Fig. 9 is $G_{s}^{\|}(z)$ at 

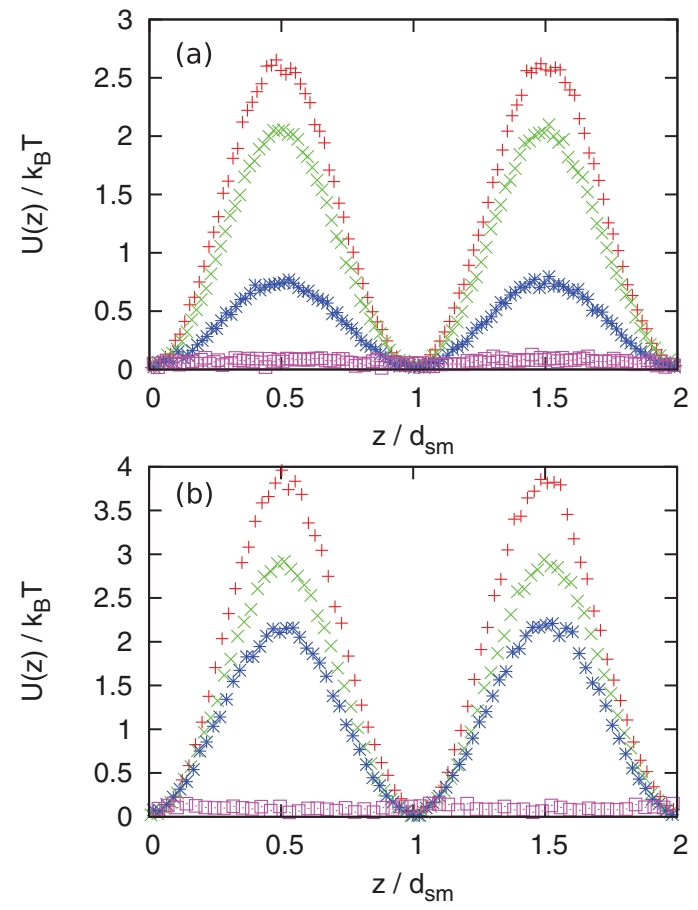

FIG. 8. Free energy barrier along the director, $U(z)$, (a) for particles with $L / L_{p}=0.31$ at $P^{*}=2.0(\square), 2.4(*), 2.5(\times)$, and $2.6(+)$, (b) and $L / L_{p}$ $=0.09$ at $P^{*}=1.3(\square), 1.6(*), 1.7(\times)$, and $1.8(+)$. Here, $d_{s m}$ is the layerto-layer spacing of the smectic phase. For both $L / L_{p}=0.31$ and 0.09 , the lowest value of pressure corresponds to a nematic phase for which $d_{s m}$ is set to one particle length.

time $t=1000 t^{*}$ and at three values of reduced pressure for the two types of particle with $L / L_{p}=0.31$ and 0.09 . As can be seen in Fig. 9, the hopping-type diffusive motion alluded to above between the layers presents itself as peaks in $G_{s}^{\|}(z)$. The peaks appear at multiples of the layer spacing, which is usually very close to one particle length. As the height of the free-energy barriers increases with increasing the pressure it becomes more difficult for particles to overcome the barriers and therefore the probability that a particle engages in an inter-layer jump decreases. On the other hand, for larger values of the barrier height particles are more confined to their layers, i.e., the width of the barriers decreases with increasing pressure (see Fig. 8), which causes the peaks in $G_{s}^{\|}$to be sharper at higher pressure.

Again, our aim is to investigate the influence of bending flexibility on the dynamics in the smectic-A phase. To do so, we cannot straightforwardly compare our simulation results for the two values of $L / L_{p}$ at the same pressure (or density), because, as we showed earlier, the pressure range at which these particles self-organize into a smectic-A phase differs. Even if there is an overlap between the two ranges, the smectic order parameters would still be different at equal pressure. Therefore, for a sensible comparison we present in Fig. 10 results of our simulations with the stiff and less stiff particles at the same value of the smectic order parameter. Shown in the inset of Fig. 10 is the self part of the Van Hove function parallel, $G_{s}^{\|}$, and in the main figure that perpendicular to the director, $G_{s}^{\perp}$, for $L / L_{p}=0.31$ and 0.09 with the same value of the smectic order parameter $\tau_{s} \approx 0.61$. Interestingly, the corre-
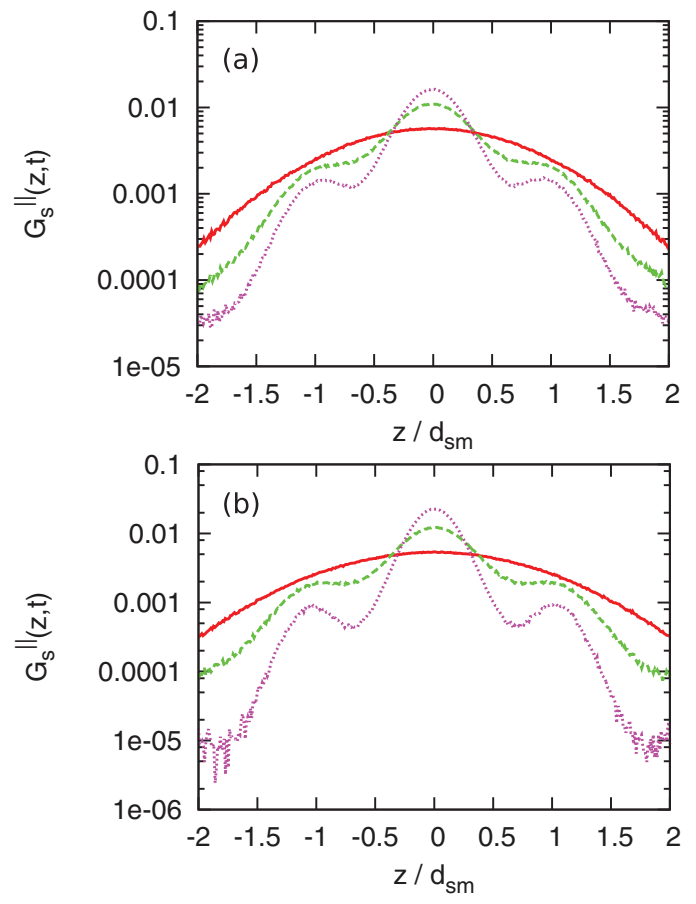

FIG. 9. Self part of the Van Hove function parallel to the director, $G_{s}^{\|}(z$, $\left.t=1000 t^{*}\right)$, as a function of the scaled distance, $z / d_{s m}$, for particles (a) with $L / L_{p}=0.31$ at $P^{*}=2.1$ (red solid line), 2.5 (green dashed line), and 2.6 (purple dotted line) and (b) with $L / L_{p}=0.09$ at $P^{*}=1.3$ (red solid line), 1.6 (green dashed line), and 1.8 (purple dotted line). Here, $d_{s m}$ is the layer-tolayer spacing of the smectic phase. For both $L / L_{p}=0.31$ and 0.09 , the lowest value of the pressure corresponds to a nematic phase state point in which case $d_{s m}$ is set equal to a single particle length.

lation functions $G_{s}^{\|}$virtually superimpose other showing that the kinetics of both types of particles along the director is very similar. Within the smectic layers, however, the more flexible particles move around much more slowly than the rigid ones do. We attribute this to particle undulation effects that cause more flexible particles to be effectively bulkier in the direction perpendicular to their principal axis, which means that they have less free space to move within a layer.

The difference between the diffusion of the particles in the directions parallel and perpendicular to the director can

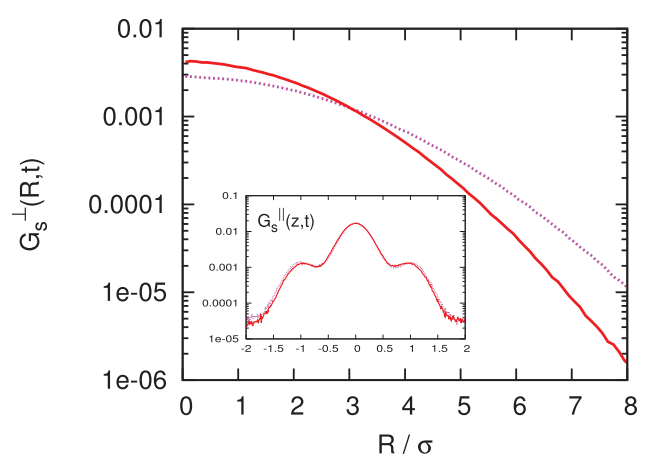

FIG. 10. Self part of the Van Hove function perpendicular, $G_{s}^{\perp}(R$, $\left.t=1000 t^{*}\right)$, and parallel, $G_{s}^{\|}\left(z, t=1000 t^{*}\right)$, (inset) to the director obtained from simulations of systems of particles with $L / L_{p}=0.31$ (red solid line) and $L / L_{p}=0.09$ (purple dotted line) at two different pressures $P^{*}=2.65$ and 1.7. The smectic order parameter for the two simulations is $\tau_{s} \approx 0.61$. 


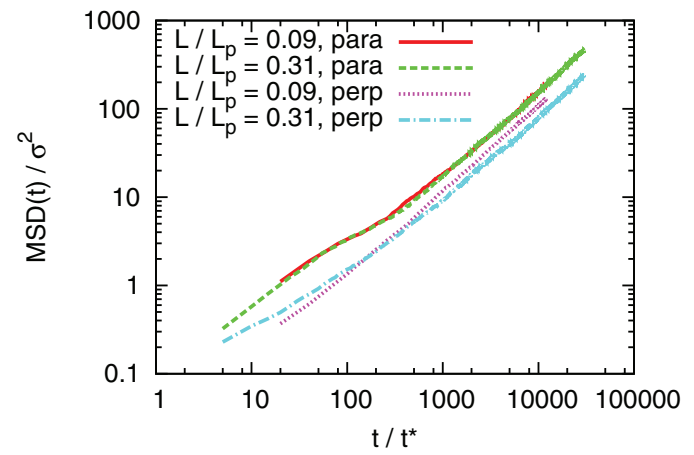

FIG. 11. Dimensionless mean square displacement, $M S D(t) / \sigma^{2}$, as a function of dimensionless time, $t / t^{*}$, obtained from simulations on particles with $L / L_{p}$ $=0.31$ and $L / L_{p}=0.09$ at identical values of the smectic order parameter $\tau_{s}$ $\approx 0.61$, corresponding to the pressures $P^{*}=2.65$ and 1.7. Here, $t^{*}$ is related to the diffusion constant of a single bead by $D_{b}=\sigma^{2} / t^{*}$ and $\sigma$ is the diameter of a bead.

also be quantified by measuring the long-time diffusion constants in these directions. To measure the diffusion constants, we first obtain the mean square displacement of the particles, $M S D(t)$, along and perpendicular to the director. Shown in Fig. 11 is the $M S D(t)$ above for particles with $L / L_{p}=0.31$ and 0.09 at the pressures $P^{*}=2.65$ and 1.7. As we expect, the $M S D(t)$ parallel to the director obtained from these simulations match at short and long time scales. There are three regimes in this direction that can be discerned from Fig. 11, a short-time regime in which the particles in the layers do not feel the presence of any of particles in the neighboring layers, an intermediate regime in which diffusion is suppressed by the self-consistent molecular field in the smectic phase and finally a long-time regime where particles jump between the smectic layers and exhibit again the usual diffusive behavior.

For the in-layer $M S D(t)$ perpendicular to the director, we identify a liquid-like behavior with two regimes: a regime where particles are caged by the neighboring particles within their layers and their diffusive motion is slowed down, and a regime in which particles hop from one cage to another resulting in a faster long-time diffusion. The short-time diffusion regime is lacking here due to the smallness of the lateral cage. Again, as we expect from what we found from the van Hove function, the long-time diffusion of more rigid particles is faster than that of the more flexible ones. In the first, "caging" regime at shorter times, however, the more flexible ones travel faster presumably because the cages formed by the more flexible particles are effectively "softer" due to particle flexing.

From the long-time behavior of $M S D(t)$, we calculate the long-time diffusion constant of particles parallel, $D_{\|}$, and perpendicular, $D_{\perp}$, to the director. Our results are shown in Fig. 12 as a function of the pressure $P^{*}$ for the two bending flexibilities corresponding to $L / L_{p}=0.31$ (green crosses) and $L / L_{p}=0.09$ (red pluses). The vertical lines in this figure indicate the approximate location of the N-Sm-A phase transition. Our results are compatible with earlier simulation studies for more rigid particles on both sides of the N-Sm-A transition. The simulations of Löwen ${ }^{27}$ on colloidal hard spherocylinders with an aspect ration of 10 agree very well with our data for $L / L_{p}=0.09$, that is to say, the values for long-time dif-

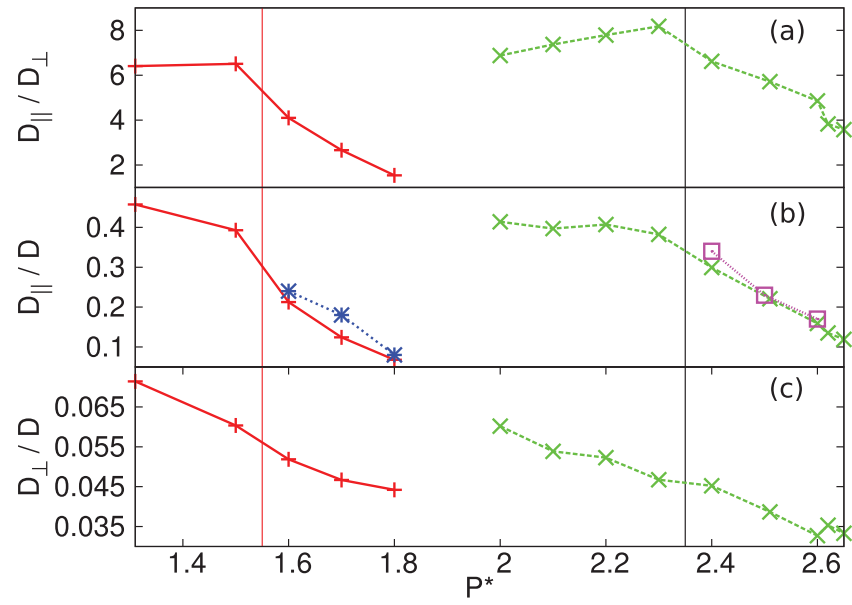

FIG. 12. (a) Ratio of long-time diffusion constants parallel, $D_{\|}$, and perpendicular, $D_{\perp}$, to the director, (b) ratio of $D_{\|}$to the single particle diffusion constant in free solution, $D$, (c) ratio of $D_{\perp}$ to $D$, all as a function of reduced pressure $P^{*}$ for the two particle flexibilities with values of $L / L_{p}=0.31$ (green crosses) and $L / L_{p}=0.09$ (red pluses). The blue and purple symbols correspond to $D_{\|}$calculated from potential barriers in the smectic phase. See the main text. The black and the red vertical lines indicate the approximate location of N-Sm-A transition for $L / L_{p}=0.31$ and 0.09 , respectively.

fusion constants at the same nematic order parameter match. Before entering the smectic-A phase the value of $D_{\|} / D_{\perp}$ in our simulations weakly increases with increasing pressure for $L / L_{p}=0.31$ whereas it is almost a constant for $L / L_{p}$ $=0.09$.

In the smectic phase, $D_{\|}$can be computed from the free energy barrier along the director, $U(z)$, by using the theoretical prediction $^{49} D_{\|}=D_{\|}^{0} /\left\langle\exp \left(-U(z) / k_{B} T\right)\right\rangle\left\langle\exp \left(U(z) / k_{B} T\right)\right\rangle$, where angular brackets indicate an average over one period of the smectic layers and we take the $D_{\|}^{0}$ as the diffusion constant in the nematic phase at a concentration close to the nematicsmectic phase transition. The values of $D_{\|}$calculated by using this method are shown in Fig. 12(b). These values are in remarkable agreement with those computed from the long-time behavior of $\operatorname{MSD}(t)$ for both the rigid and the more flexible particles.

We furthermore find that for the two particle flexibilities the values of $D_{\|} / D, D_{\perp} / D$, and $D_{\|} / D_{\perp}$ at the nematicsmectic transition do not change significantly with flexibility, and $D_{\|} / D_{\perp}$ decreases with increasing density and entering the Sm-A phase. Our finding is in contrast with the experimental observations on wild-type and mutant $f d$ virus particles, where $D_{\|} / D_{\perp}$ increases with density (and hence pressure) after entering the smectic-A phase for the more flexible wildtype $f d$ virus particles. The discrepancy might be due to the small aspect ratio of our particles compared to that of $f d$ virus or the impact of hydrodynamic interactions that we ignore completely. ${ }^{26}$

\section{CONCLUSIONS}

We carried out molecular and Brownian dynamics simulations, and studied the influence of particle bending flexibility on the equilibrium properties and dynamics of dispersions of filamentous particles at different densities. Motivated by recent experiments on $f d$ virus particles, we did our 
simulations for persistence lengths corresponding to those of wild-type and mutant $f d$ virus particles. By measuring the nematic, smectic, and bond-orientational order parameters, we located the density at which our particles self-organize into nematic, smectic-A, smectic-B, and/or crystal phases.

In agreement with theoretical predictions, we find that the N-Sm-A phase transition density is shifted towards larger values for the more flexible particles. We also find that particle flexibility changes the smectic layer-to-layer distance as a function of density. For the more rigid particles, it decreases monotonically with increasing density whereas for the more flexible ones it first increases and after that decreases. We attribute this to thermal undulations of the more flexible particles that are suppressed at higher densities. Moreover, the more flexible particles at sufficiently high densities selforganize into the smectic-B phase in which particles within the smectic layers exhibit hexagonal ordering yet the corresponding hexagonal lattice is displaced randomly from one layer to another. For the more rigid particles, the hexagonal lattice of each layer is almost aligned with the next one and in this case we cannot distinguish between smectic-B and crystal phases.

Our simulations on the dynamics of these particles in the smectic-A phase show that both types of particle exhibit a hopping-type diffusion between the smectic layers. We show that at densities that both types of particle have the same value of the smectic order parameter, their diffusion along the director is very similar but more flexible particles move slower in the direction perpendicular to it. We also see this in the longtime behavior of the mean-square displacement of the particles in these two simulations. At relatively short time scales, where caging of particles by neighbors predominates the kinetics, the more flexible particles move about faster. We attribute this to the particle flexing that presumably cause the cages formed by neighbors of each particle to be effectively "softer."

Our results on the diffusion of particles shows that particle flexibility does not change the diffusive behavior on both sides of the N-Sm-A transition significantly and for the both stiff and more flexible particles the ratio of $D_{\|}$to $D_{\perp}$ decreases with entering the Sm-A phase.

\section{ACKNOWLEDGMENTS}

The work of S.N. forms part of the research program of the Dutch Polymer Institute (DPI, Project No. 698).

${ }^{1} \mathrm{P}$. Chaikin and T. Lu bensky, Principles of Condensed Matter Physics (Cambridge University Press, Cambridge, 1994).

${ }^{2}$ Z. Dogic and S. Fraden, Langmuir 16, 7820 (2000).

${ }^{3}$ F. Tombolato, A. Ferrarini, and E. Grelet, Phys. Rev. Lett. 96, 258302 (2006).
${ }^{4}$ R. B. Meyer, in Dynamics and Patterns in Complex Fluids, edited by A. Onuki and K. Kawasaki (Springer, Berlin, 1990), Vol. 52.

${ }^{5}$ F. Livolant, Physica A 176, 117 (1991).

${ }^{6}$ L. Onsager, Ann. N.Y. Acad. Sci. 51, 627 (1949).

${ }^{7}$ D. Frenkel, H. N. W. Lekkerkerker, and A. Stroobants, Nature (London) 332, 822 (1988).

${ }^{8}$ P. Bolhuis and D. Frenkel, J. Chem. Phys. 106, 666 (1997).

${ }^{9}$ D. Frenkel and B. M. Mulder, Mol. Phys. 55, 1171 (1985).

${ }^{10}$ A. Stroobants, H. N. W. Lekkerkerker, and D. Frenkel, Phys. Rev. Lett. 57, 1452 (1986).

${ }^{11}$ S. C. McGrother, D. C. Williamson, and G. Jackson, J. Chem. Phys. 104, 6755 (1996).

${ }^{12}$ A. Poniewierski and R. Hołyst, Phys. Rev. Lett. 61, 2461 (1988).

${ }^{13}$ B. Mulder, Phys. Rev. A 35, 3095 (1987).

${ }^{14}$ S. M. Yu, V. P. Conticello, G. Zhang, C. Kayser, M. J. Fournier, T. L. Mason, and D. A. Tirrell, Nature (London) 389, 167 (1997).

${ }^{15}$ A. Kuijk, A. van Blaaderen, and A. Imhof, J. Am. Chem. Soc. 133, 2346 (2011).

${ }^{16}$ X. Wen, R. B. Meyer, and D. L. D. Caspar, Phys. Rev. Lett. 63, 2760 (1989).

${ }^{17}$ Z. Dogic and S. Fraden, Phys. Rev. Lett. 78, 2417 (1997).

${ }^{18}$ P. van der Schoot, J. Phys. II (France) 6, 1557 (1996).

${ }^{19}$ P. Bladon and D. Frenkel, J. Phys.: Condens. Matter 8, 9445 (1996).

${ }^{20}$ R. C. Hidalgo, D. E. Sullivan, and J. Z. Y. Chen, Phys. Rev. E 71, 041804 (2005).

${ }^{21}$ A. V. Tkachenko, Phys. Rev. Lett. 77, 4218 (1996).

${ }^{22}$ Z. Dogic, J. Zhang, A. W. C. Lau, H. Aranda-Espinoza, P. Dalhaimer, D. E. Discher, P. A. Janmey, R. D. Kamien, T. C. Lubensky, and A. G. Yodh, Phys. Rev. Lett. 92, 125503 (2004).

${ }^{23}$ J. Käs, H. Strey, J. Tang, D. Finger, R. Ezzell, E. Sackmann, and P. Janmey, Biophys. J. 70, 609 (1996).

${ }^{24}$ H. Maeda and Y. Maeda, Phys. Rev. Lett. 90, 018303 (2003).

${ }^{25}$ M. P. Lettinga, E. Barry, and Z. Dogic, Europhys. Lett. 71, 692 (2005).

${ }^{26}$ M. P. Lettinga, J. K. G. Dhont, Z. Zhang, S. Messlinger, and G. Gompper, Soft Matter 6, 4556 (2010).

${ }^{27}$ H. Löwen, Phys. Rev. E 59, 1989 (1999).

${ }^{28}$ M. P. Allen, Phys. Rev. Lett. 65, 2881 (1990).

${ }^{29}$ M. P. Lettinga and E. Grelet, Phys. Rev. Lett. 99, 197802 (2007).

${ }^{30}$ E. Grelet, M. P. Lettinga, M. Bier, R. van Roij, and P. van der Schoot, J. Phys.: Condens. Matter 20, 494213 (2008).

${ }^{31}$ M. Bier, R. van Roij, M. Dijkstra, and P. van der Schoot, Phys. Rev. Lett. 101, 215901 (2008).

${ }^{32}$ A. Kuijk, D. Byelov, A. Petukhov, A. van Blaaderen, and A. Imhof, Faraday Discuss. 159, 181 (2012).

${ }^{33}$ R. Matena, M. Dijkstra, and A. Patti, Phys. Rev. E 81, 021704 (2010).

${ }^{34}$ A. Patti, D. El Masri, R. van Roij, and M. Dijkstra, J. Chem. Phys. 132, 224907 (2010).

${ }^{35}$ A. Patti, D. El Masri, R. van Roij, and M. Dijkstra, Phys. Rev. Lett. 103, 248304 (2009).

${ }^{36}$ G. Cinacchi and L. De Gaetani, Phys. Rev. E 79, 011706 (2009).

${ }^{37}$ E. Barry, D. Beller, and Z. Dogic, Soft Matter 5, 2563 (2009).

${ }^{38}$ E. Pouget, E. Grelet, and M. P. Lettinga, Phys. Rev. E 84, 041704 (2011).

${ }^{39}$ S. Plimpton, J. Comput. Phys. 117, 1 (1995).

${ }^{40}$ A. R. Khokhlov and A. N. Semenov, Physica A 108, 546 (1981).

${ }^{41}$ A. R. Khokhlov and A. N. Semenov, Physica A 112, 605 (1982).

${ }^{42}$ E. Grelet, Phys. Rev. Lett. 100, 168301 (2008).

${ }^{43}$ S. Naderi, E. Pouget, P. Ballesta, P. van der Schoot, M. P. Lettinga, and E. Grelet, Phys. Rev. Lett. 111, 037801 (2013).

${ }^{44} \mathrm{M}$. Doi and S. F. Edwards, The Theory of Polymer Dynamics (Oxford University Press, USA, 1988), Vol. 73.

${ }^{45}$ E. Grelet, Phys. Rev. X 4, 021053 (2014).

${ }^{46}$ J. A. C. Veerman and D. Frenkel, Phys. Rev. A 43, 4334 (1991).

${ }^{47}$ G. Cinacchi and L. De Gaetani, Phys. Rev. E 77, 051705 (2008).

${ }^{48}$ E. Grelet, personal communication (2013).

${ }^{49}$ R. Festa and E. d'Agliano, Physica A 90, 229 (1978). 